\title{
Décrypter le théâtre de l'absurde ne suffit plus : retrouver le chemin de la dissidence
}

La pré-conférence des parties à la convention sur la diversité biologique (COP 15) et la COP 26 de la convention-cadre des Nations unies sur les changements climatiques viennent de s'achever. La revue Natures Sciences Sociétés a été créée en 1993 au lendemain du Sommet de la Terre de Rio où ont été signées ces deux conventions. Depuis, une grande attention a été portée aux négociations censées définir la gouvernance mondiale du développement durable ${ }^{1}$.

Faut-il parler de fatigue, de découragement ou de colère quand il s'agit aujourd'hui de rendre compte de ces négociations? Les menaces sur l'environnement et l'urgence à agir sont de mieux en mieux documentées par les scientifiques ${ }^{2}$ et de plus en plus ressenties par les citoyens, mais rien n'y fait: ces conférences rejouent le même spectacle.

Le diagnostic sur l'état de la planète et les blocages à lever étaient déjà présents à la première conférence de Stockholm de 1972, il y a 50 ans... Le rapport Meadows, qui n'intégrait qu'à la marge le danger climatique, modélisait les limites de la croissance. La population mondiale était deux fois moins importante qu'aujourd'hui. Les pays du Sud se regroupaient en coalition de pays non alignés et Indira Gandhi dénonçait: «La pauvreté et le besoin ne sont-ils pas les plus grands pollueurs?». Le premier principe de la déclaration de Stockholm reste d'une totale actualité: «[...] les politiques qui encouragent ou qui perpétuent l'apartheid, la ségrégation raciale, la discrimination, les formes, coloniales et autres, d'oppression et de domination étrangères sont condamnées et doivent être éliminées ».

\footnotetext{
1 Voir par exemple les numéros spéciaux de NSS: La biodiversité: un problème d'environnement global (NSS, 1998, 6, 1; NSS, 1998, 6, 2); Les enjeux de la conférence de Paris, penser autrement la question climatique (NSS, 2015, 23, Sup.).

${ }^{2}$ Voir les rapports de l'IPBES: The global assessment report on biodiversity and ecosystem services of the Intergovern mental Science-Policy Platform on Biodiversity and Ecosys tem Services (2019); de l'IPCC, AR6 Climate Change 2021: The Physical Science Basis (2021); et le rapport conjoint IPBES-IPCC, Co-sponsored workshop report on biodiversity and climate change (2021).
}

Hélas, le néolibéralisme de Ronald Reagan et de Margaret Thatcher, puis la chute du mur de Berlin en 1989 et « la fin de l'histoire» ont changé la donne. C'est donc sur la croyance dans le pouvoir régulateur du marché qu'ont été rédigées les conventions de Rio. La création des marchandises, crédits carbone et ressources génétiques, devaient résoudre la question du changement climatique, présenté comme une pollution, et l'érosion de la biodiversité, présentée comme un défaut de droits de propriété. Les pays du Sud se sont résolus dans un premier temps à adhérer à ces solutions marchandes en espérant quelques retours financiers et considérations politiques, jusqu'à de profondes désillusions.

On ne s'étonnera pas de retrouver aujourd'hui les mêmes sujets de blocage des négociations. Tout d'abord la question du financement, qui peut s'analyser comme une question de confiance: le Nord n'a pas confiance dans les engagements des pays du Sud et multiplie des exigences de contrôle, de reporting, ces mécanismes de transparence et de redevabilité. Les pays du Sud ne voient pas pourquoi ils accepteraient ces comptabilités tatillonnes alors que les pays du Nord sont incapables de réunir les sommes promises lors de la COP de Copenhague en 2009, soit 100 milliards US\$ par an pour le climat, dix fois moins que ce qu'il faudrait pour arriver à amorcer une économie décarbonée... Le Sud réclame plus de moyens, y compris pour l'adaptation et pour les pertes et dommages dus aux catastrophes climatiques, et non seulement pour réduire les émissions. Le cadre mondial de la biodiversité post 2020 qui sera discuté en avril 2022 à Kunming lors de la COP 15, vise une réorientation des subventions néfastes ( 500 milliards) et une augmentation de 200 milliards, dont 10 milliards pour les pays en développement...

Pour le climat, les négociations sont âpres autour de l'article 6 de l'accord de Paris concernant les mécanismes de marché des émissions de gaz à effet de serre. Quand un pays du Nord achète des crédits carbone à un pays du Sud qui a limité ses émissions, à quel pays doiton imputer la baisse des émissions ?... Ces doubles comptes empoisonnent les débats.

Pour la biodiversité, le problème porte sur l'inclusion des séquences génétiques, digital sequence information 
(DSI), dans le champ de la convention. Ces séquences que l'on trouve en accès libre dans les banques de gènes occidentales échappent au principe d'accès et de partage des avantages du protocole de Nagoya, aujourd'hui ratifié par 132 pays $^{3}$. Ce protocole encadre l'accès à la ressource génétique in situ ou ex situ, mais non à la ressource une fois séquencée, in silico. Les dénonciations de pillage du Tiers monde et de biopiraterie ressurgissent de plus belle.

Ces points d'achoppement sont complexes, techniques, chronophages, et semblent ignorer l'urgence à agir. Par leur abstraction, ils peuvent sembler détachés des enjeux, mais ils renvoient aux débats de Stockholm: quand les pays industrialisés veulent résoudre les problèmes d'environnement par la technique, la science et le marché, les pays du Sud dénoncent les inégalités géopolitiques, la colonisation, la mondialisation au service des intérêts des pays du Nord.

Il est cependant trop facile de s'en tenir à la critique de ces jeux de postures propres aux négociations, alors que les gouvernements poursuivent leur course effrénée à la croissance. Les plans de relance post-Covid-19 visent à redynamiser la consommation et l'industrialisation, avec force recours aux matériaux et à l'énergie. Les sommes consacrées à la biodiversité dans ces plans sont dérisoires.

Si les chercheurs oscillent entre découragement et colère, entre culpabilité de n'avoir pas su se faire entendre ou d'avoir laissé faire, quel est alors le rôle de Natures Sciences Sociétés? Fondée sur l'engagement pour l'interdisciplinarité, NSS vise à comprendre les défis de la transition environnementale, à rompre avec la pensée réductionniste et rationaliste des disciplines et des savoirs fondés sur des évidences, pour aborder la complexité du monde, l'incertitude, les conflits de valeurs.

Sans doute l'institutionnalisation de l'interdisciplinarité et la généralisation des recherches participatives qui sous-tendent les articles publiés, évoquées lors de la journée de l'association NSS-Dialogues consacrée à l'actualité de la question interdisciplinaire le 14 octobre dernier, ont poussé à une sorte de normalisation et ont estompé l'engagement politique initial de la revue. Il s'agit aujourd'hui de le réaffirmer et de retrouver le chemin de la dissidence. Ces négociations qui patinent nous y exhortent.

\footnotetext{
3 Voir: Aubertin C., Nivart A. (Eds), 2021. La nature en partage : autour du protocole de Nagoya. Marseille, Paris, IRD éditions/MNHN.
} 